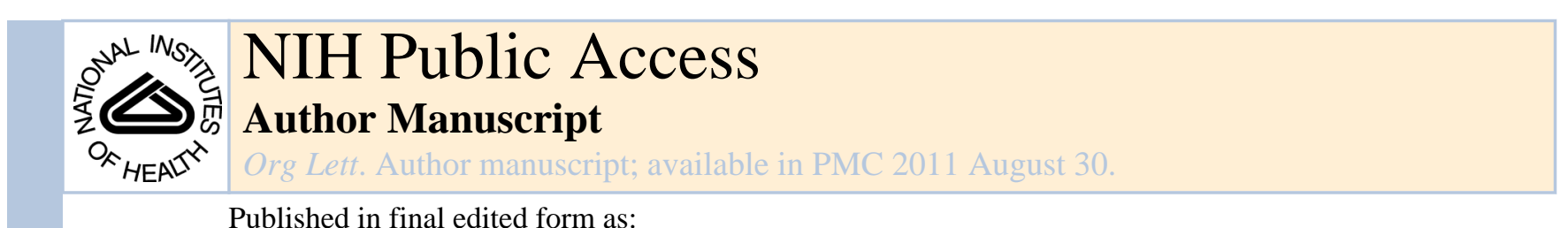

Published in final edited form as:

Org Lett. 2006 April 13; 8(8): 1513-1516. doi:10.1021/ol052862g.

\title{
Torsional Steering Controls the Stereoselectivity of Epoxidation in the Guanacastepene A Synthesis
}

\author{
Paul Ha-Yeon Cheong, Heedong Yun ${ }^{\dagger}$, Samuel J. Danishefsky ${ }^{\dagger}$, and K. N. Houk \\ Department of Chemistry and Biochemistry, University of California, Los Angeles, CA \\ 90095-1569 \\ †Department of Chemistry, Columbia University, 3000 Broadway, New York, NY 10027
}

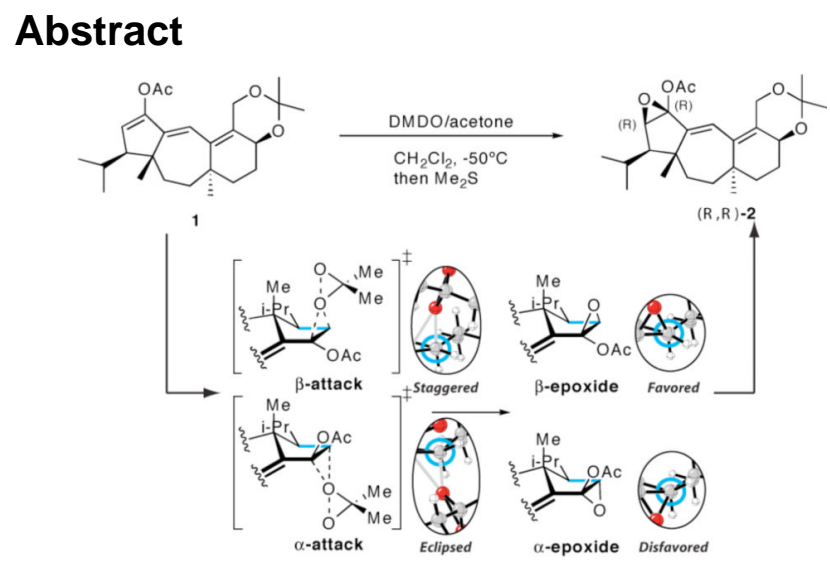

The stereoselectivity of the key epoxidation step in the synthesis of Guanacastepene A is shown to be controlled by torsional steering. In this particular epoxidation reaction, the transition structure energetic difference is enhanced by the great asynchronicity of the forming $\mathrm{C}-\mathrm{O}$ bonds that intensifies the torsional interactions.

The stereoselective epoxidation of the Guanacastepene A precursor $\mathbf{1}$ constitutes a key step which provides the correct stereochemistry of the $\beta$-acetoxy ketone, as shown above. ${ }^{1}$ Cursory inspection of the structure suggests that attack should occur on the $\alpha$-face, yet exclusive formation of the $\beta$-epoxide was observed experimentally. The crystal structure of the hydroxyketone formed from $\mathbf{2}$ shows that the isopropyl group is in equatorial position, imparting little steric differentiation between the $\alpha$ - and $\beta$-faces. ${ }^{\text {lc }}$ The computational study described here reveals that the origin of stereoselective $\beta$-attack in this epoxidation is a strong torsional control of stereoselectivity that we have named torsional steering. ${ }^{2}$

The computed epoxidation transition structures of a model alkene containing all the substituents of the cyclopentene ring of guanacastepene precursor $\mathbf{1}$, computed with density functional theory (B3LYP/6-31G*), ${ }^{3}$ are shown in Figure $1 .{ }^{4}$ The $\alpha$-epoxidation transition structure TS-(S,S)-3, with the dimethyldioxirane (DMDO) approaching from the sterically less hindered face of the cyclopentene ring is $4 \mathrm{kcal} / \mathrm{mol}$ higher in energy than the analogous

Correspondence to: K. N. Houk.

houk@chem.ucla.edu .

Supporting Information Available. Cartesian and internal redundant coordinates, energies, thermodynamic corrections for all reported structures, full authorship of reference 3 . 
$\beta$ - epoxidation process TS- $(\boldsymbol{R}, \boldsymbol{R})-\mathbf{3}$, in agreement with experimental observations that only the $\beta$-epoxide is formed.

Both transition structures are very asynchronous; one of the forming $\mathrm{C}$-O bond is $\sim 1.8 \AA$, while the other forming $\mathrm{C}-\mathrm{O}$ bond length is $\sim 2.4 \AA$. This high asynchronicity is the result of electron donation from the acetoxy group and the conjugated alkene.

The inherent stability difference between the transition structures is due to the difference in torsional strain between bonds linking the forming epoxide to the rest of the skeleton, as shown in the insets Newman projections in Figure 1. The $\alpha$-epoxidation transition structure TS- $(\boldsymbol{S}, \boldsymbol{S})-\mathbf{3}$ exhibits substantial eclipsing, in contrast to the substantially more staggered $\beta$ epoxidation transition structure TS-(R,R)-3. Such torsional control of electrophilic or nucleophilic reactions has been observed in many reactions. ${ }^{2}$

The chair product $(\boldsymbol{S}, \boldsymbol{S}) \mathbf{- 4}$, which arises from the $\alpha$-epoxidation, is also higher in energy by 3 $\mathrm{kcal} / \mathrm{mol}$ than the boat product $(\boldsymbol{R}, \boldsymbol{R})-\mathbf{4}$, from $\beta$-epoxidation. Bicyclo[3.1.0] hexane systems are known to prefer the boat conformation over the chair due to torsional interactions with the bridgeheads. ${ }^{5}$ In the case of parent 6-oxabicyclo[3.1.0] hexane, this preference is computed to be $4.0 \mathrm{kcal} / \mathrm{mol} .{ }^{5 \mathrm{~d}}$ As shown in the corresponding insets, the difference between the eclipsing in $(\boldsymbol{S}, \boldsymbol{S})-\mathbf{4}$ and $(\boldsymbol{R}, \boldsymbol{R})-\mathbf{4}$ is reduced as compared to the transition state.

It is noteworthy that the energy difference between the transition structures is greater than the energy difference between the products by $\sim 1 \mathrm{kcal} / \mathrm{mol}$. This is unexpected, given that the torsional interactions that emerge in the products should be stronger than in the transition states that precede them. This enhancement of stereoselectivity comes from the great asynchronicity of the transition structures - the lengthening of one of the C-O bonds intensifies the eclipsing or staggering effect. In the products, the epoxide forces the C-O bond to bend in such a way that the analogous conformational effects are somewhat ameliorated.

In order to assess the effect that the rest of $\mathbf{1}$ may have on the transition structures, activation barriers for the actual guanacastepene precursor were also computed with the ONIOM method. ${ }^{3}$ Density functional theory (B3LYP/6-31G*) was used for the cyclopentene ring of interest, while PM3 semi-empirical method was used for the rest of the target molecule, as shown in Scheme 1.

The $\beta$-epoxidation transition structure TS- $(\boldsymbol{R}, \boldsymbol{R})-\mathbf{5}$ is shown to be more stable by $\sim 2.5 \mathrm{kcal} /$ mol than the analogous $\alpha$-epoxidation transition structure TS-(S,S)-5. The slight lowering of the energetic difference is attributed to stabilizing $\mathrm{CH}^{\delta+} \_{ }_{-}-\mathrm{O}$ electrostatic interactions between the substituents of the cyclopentene ring and the approaching DMDO in TS$(S, S)-5$.

We have also investigated the effect of entropy and solvation on the stereoselectivity of this reaction. In all cases, the $\beta$-epoxidation transition structures were more stable than the analogous $\alpha$-epoxidation transition structures. The free energy difference for the model transition structure TS-(S,S)-3 and TS-(R,R)-3 is $3.9 \mathrm{kcal} / \mathrm{mol}$, while the analogous difference between the ONIOM transition structures TS- $(\boldsymbol{S}, \boldsymbol{S})-\mathbf{5}$ and TS- $(\boldsymbol{R}, \boldsymbol{R})-\mathbf{5}$ is $2.6 \mathrm{kcal} /$ mol. Single point PCM solvation energy calculations have been performed using the HF/ $6-31+\mathrm{G}(\mathrm{d}, \mathrm{p})$ basis set and $\mathrm{UAKS}$ radii with $\mathrm{CHCl}_{2}$ as the solvent. Solvation energy decreases the computed stereoselectivity by $2.6 \mathrm{kcal} / \mathrm{mol}$ for the model transition structures and $1.6 \mathrm{kcal} / \mathrm{mol}$ for the ONIOM transition structures. This decrease in computed stereoselectivity is the result of greater electrostatic stabilization of the later, hence more zwitterionic $\alpha$-epoxidation transition structures. In the products $(\boldsymbol{S}, \boldsymbol{S})-\mathbf{4}$ and $(\boldsymbol{R}, \boldsymbol{R})-\mathbf{4}$ where 
the difference in zwitterionic character should be minimal, the change in stereoselectivity is minimal $(0.8 \mathrm{kcal} / \mathrm{mol})$.

Torsional steering, as the effect has been called, is a general factor involved in additions of nucleophiles, radicals, and electrophiles to multiple bonds, including carbonyls, imines and alkenes. ${ }^{2}$ The importance of such an effect was first identified by Felkin in the discussion of nucleophilic additions to cyclohexanones, ${ }^{6}$ but it is now clear that it governs stereoselectivity in a wide variety of situations. ${ }^{2}$ It can actually be identified in the conformation of the alkene reactant, shown in Figure 2. The effect influences the relative energies of diastereomeric conformations that involve torsional factors not present in the reactants, but which become significant in the products of the reaction. This effect has been reported earlier, ${ }^{2}$ but the current example in the guanacastepene synthesis is especially striking.

\section{Supplementary Material}

Refer to Web version on PubMed Central for supplementary material.

\section{Acknowledgments}

We are grateful to the National Institute of General Medical Sciences, National Institutes of Health for financial support of this research. This research was facilitated through the Partnerships for Advanced Computational Infrastructure (PACI) through the support of the National Science Foundation. The computations were performed on the National Science Foundation Terascale Computing System at the Pittsburgh Supercomputing Center (PSC) and on the UCLA Academic Technology Services (ATS) Hoffman Beowulf cluster.

\section{References}

1. a Lin S, Dudley GB, Tan DS, Danishefsky SJ. Angew. Chem. Int. Ed. 2002; 41:2188.b Mandal M, Danishefsky SJ. Tetrahedron Lett. 2004; 45:3831.c Mandal M, Yun H, Dudley GB, Lin S, Tan DS, Danishefsky SJ. J. Org. Chem. 2005 ASAP.

2. a Rondan NG, Paddon-Row MN, Caramella P, Mareda J, Mueller PH, Houk KN. J. Am. Chem. Soc. 1982; 104:4974.b Houk KN, Tucker JA, Dorigo AE. Acc. Chem. Res. 1990; 23:107.c Martinelli MJ, Peterson BC, Khau VV, Hutchinson DR, Leanna MR, Audia JE, Droste JJ, Wu Y-D, Houk KN. J. Org. Chem. 1994; 59:2204.d Lucero MJ, Houk KN. J. Org. Chem. 1998; 63:6973. [PubMed: 11672319] e Wu Y-D, Houk KN, Trost BM. J. Am. Chem. Soc. 1987; 109:5560.Mukherjee D, Wu Y-D, Fronczek FR, Houk KN. J. Am. Chem. Soc. 1988; 110:3328.f Ando K, Green NS, Li Y, Houk KN. J. Am. Chem. Soc. 1999; 121:5334.g Brown FK, Houk KN. J. Am. Chem. Soc. 1985; 107:1971.h Houk KN, Paddon-Row MN, Rondan NG, Wu Y-D, Brown FK, Spellmeyer DC, Metz JT, Li Y, Loncharich RJ. Science. 1986; 231:1108. [PubMed: 3945819]

3. Frisch MJ, et al. , Gaussian03. See supporting information for full authorship of Gaussian.

4. a Houk KN, Liu J, DeMello NC, Condroski KR. J. Am. Chem. Soc. 1997; 119:10147.b Jenson C, Liu J, Houk KN, Jorgensen WL. J. Am. Chem. Soc. 1997; 119:12982.c Singleton A, Merrigan SR, Liu J, Houk KN. J. Am. Chem. Soc. 1997; 119:3385.d Washington I, Houk KN. J. Am. Chem. Soc. 2000; 122:2948.

5. Grostic MF, Duchamp DJ, Chidester CG. J. Org. Chem. 1971; 36:2929.b Cook RL, Malloy TB Jr. J. Am. Chem. Soc. 1974; 96:1703.c Mastryukov VS, Osina EL, Vilkov LV, Hilderbrandt RL. J. Am. Chem. Soc. 1977; 99:6855. d Kang P, Choo J, Jeong M, Kwon Y. J. Mol. Struct. 2000; 519:75.e Cheong PH-Y, Houk KN, Warrier JS, Hanessian S. Adv. Synth. Cat. 2004; 346:1111. Computed at B3LYP/6-31G** level of theory:

6. a Cherest M, Felkin H. Tetrahedron Lett. 1968:2205.b Cherest M, Felkin H, Frajerman C. Tetrahedron Lett. 1971:379.c Cherest M, Felkin H. Tetrahedron Lett. 1971:383. 

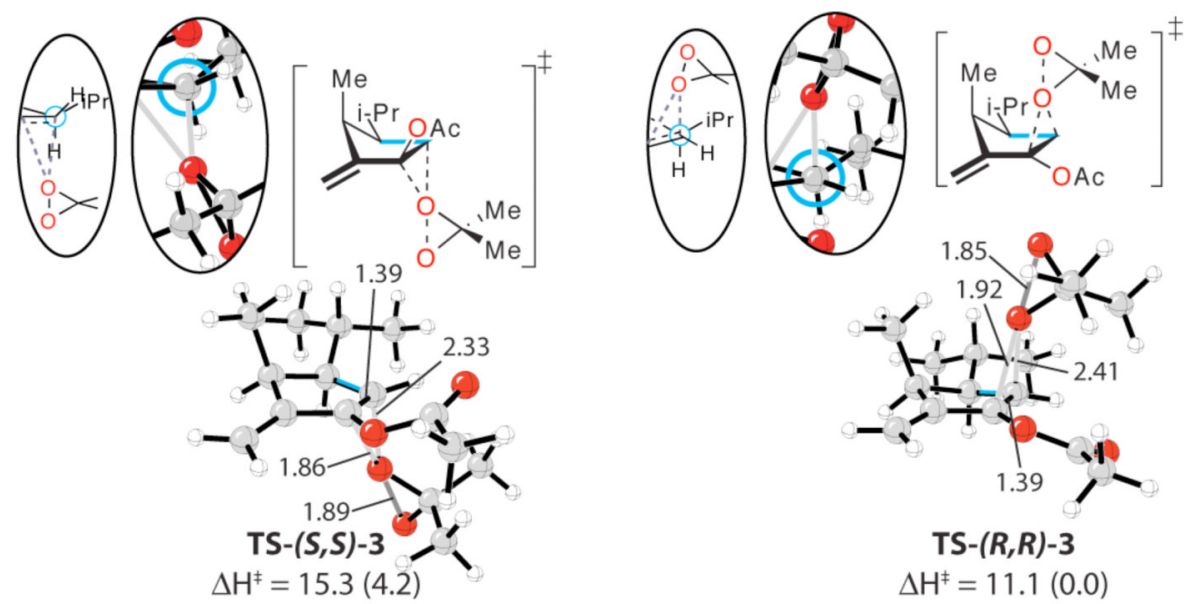

TS- $(R, R)-3$

$\Delta \mathrm{H}^{\ddagger}=11.1(0.0)$
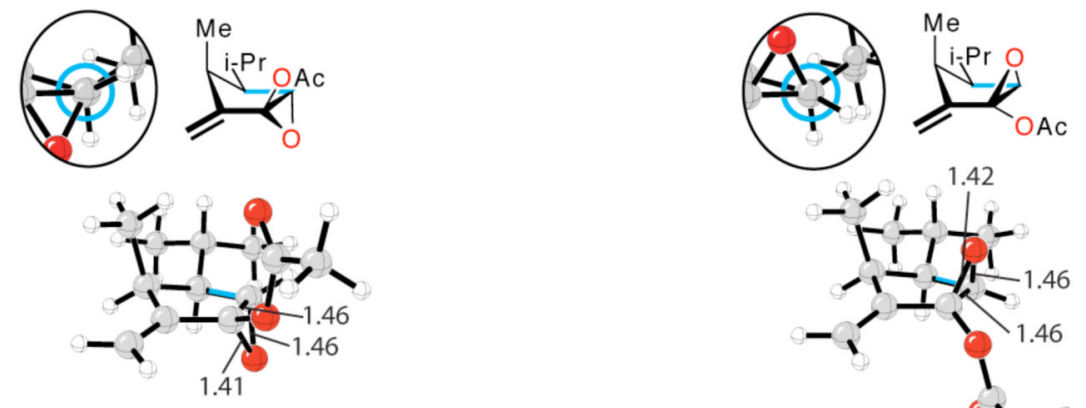

(S,S)-4

$\Delta \mathrm{H}=-50.3(3.2)$
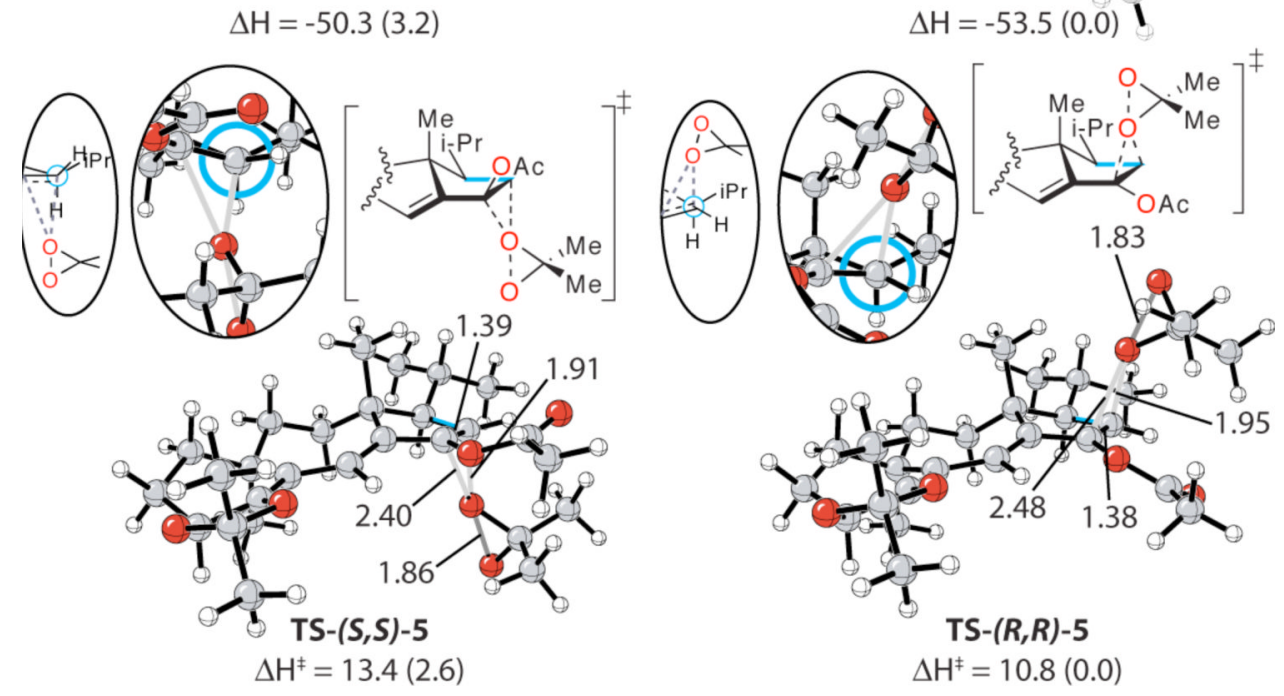

Figure 1.

Model transition structures (TS- $(\boldsymbol{S}, \boldsymbol{S})-\mathbf{3}$ and TS- $(\boldsymbol{R}, \boldsymbol{R})-\mathbf{3})$, model $\alpha$ - and $\beta$-epoxide products $((S, S)-4$ and $(\boldsymbol{R}, \boldsymbol{R})-\mathbf{4})$, and full transition structures (TS- $(S, S)-5$ and TS- $(R, R)-5)$. The torsion of interest is highlighted in blue in each computed structure, and the corresponding Newman projections are shown in the oval insets. Energy values are in $\mathrm{kcal} / \mathrm{mol}$. Values enclosed in parenthesis are relative energies in $\mathrm{kcal} / \mathrm{mol}$. 

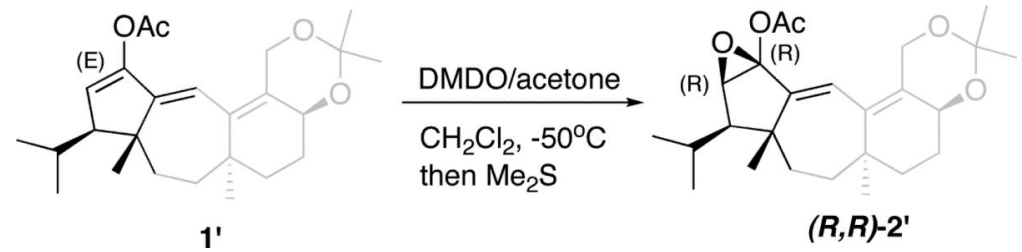

Scheme 1.

ONIOM partition as described in text. Black region was modeled with B3LYP/6-31G*, while the grey region was modeled by PM3. 

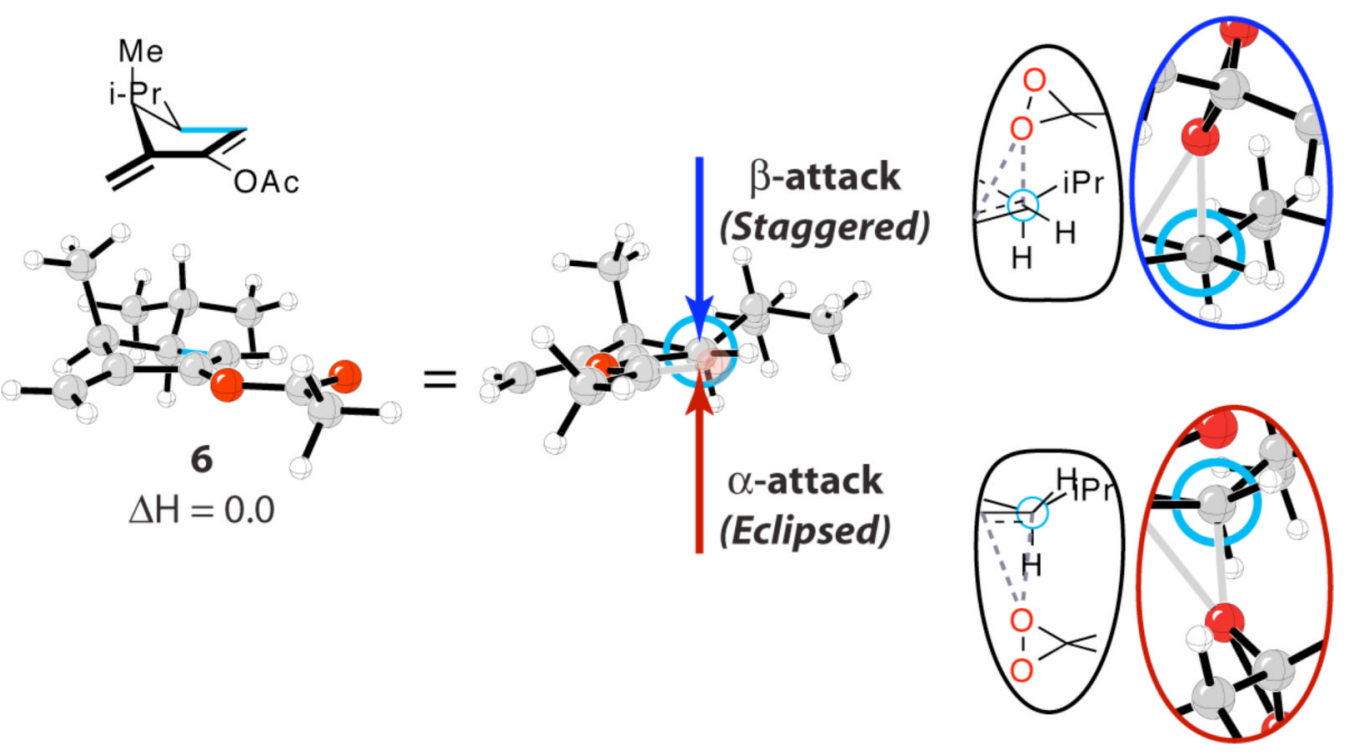

Figure 2.

Reactant structure $\mathbf{6}$ and Newman projection that illustrate the resultant torsional effects for the $\alpha$ - and $\beta$-epoxidation transition states provided in the insets. Acetoxy oxygen has been rendered transparent in the Newman projection for clarity. The torsion of interest is highlighted in blue in reactant structure 6. 